\title{
Control Strategies for Integration of Electric Motor Assist and Functional Electrical Stimulation in Paraplegic Cycling: Utility for Exercise Testing and Mobile Cycling
}

\author{
Kenneth J. Hunt, Member, IEEE, Barry Stone, Nils-Otto Negård, Thomas Schauer, Matthew H. Fraser, \\ Andrew J. Cathcart, Chiara Ferrario, Susan A. Ward, and Stan Grant
}

\begin{abstract}
Aim: The aim of this study was to investigate feedback control strategies for integration of electric motor assist and functional electrical stimulation (FES) for paraplegic cycling, with particular focus on development of a testbed for exercise testing in FES cycling, in which both cycling cadence and workrate are simultaneously well controlled and contemporary physiological measures of exercise performance derived. A second aim was to investigate the possible benefits of the approach for mobile, recreational cycling.

Methods: A recumbent tricycle with an auxiliary electric motor is used, which is adapted for paraplegic users, and instrumented for stimulation control. We propose a novel integrated control strategy which simultaneously provides feedback control of leg power output (via automatic adjustment of stimulation intensity) and cycling cadence (via electric motor control). Both loops are designed using system identification and analytical (model-based) feedback design methods. Ventilatory and pulmonary gas exchange response profiles are derived using a portable system for real-time breath-by-breath acquisition.

Results: We provide indicative results from one paraplegic subject in which a series of feedback-control tests illustrate accurate control of cycling cadence, leg power control, and external disturbance rejection. We also provide physiological response profiles from a submaximal exercise step test and a maximal incremental exercise test, as facilitated by the control strategy.
\end{abstract}

Manuscript received September 6, 2002; revised May 21, 2003. This work was supported by the Engineering and Physical Sciences Research Council under Grant GR/M94717 and Grant GR/R92462 (Feedback Control Strategies for Integration of Human and Motor Power in Paraplegic Cycling) and by the INSPIRE Foundation (A Pilot Study of Lower-limb FES Cycling in Paraplegia).

K. J. Hunt is with the Centre for Rehabilitation Engineering, Department of Mechanical Engineering, University of Glasgow, Glasgow G12 8QQ, U.K the Queen Elizabeth National Spinal Injuries Unit, Southern General Hospital NHS Trust, Glasgow G51 4TF, U.K., and the Centre for Exercise Science and Medicine, Faculty of Biomedical and Life Sciences, University of Glasgow, Glasgow G12 8QQ, U.K. (e-mail: k.hunt@mech.gla.ac.uk).

B. Stone and C. Ferrario are with the Centre for Rehabilitation Engineering, Department of Mechanical Engineering, University of Glasgow, Glasgow G12 8QQ, U.K.

N.-O. Negård and T Schauer are with Centre for Rehabilitation Engineering, Department of Mechanical Engineering, University of Glasgow, Glasgow G12 $8 Q Q$, U.K, and also with the Systems and Control Theory Group, Max Planck Institute for Dynamics of Complex Technical Systems, D-39106 Magdeburg, Germany.

M. H. Fraser is with the Queen Elizabeth National Spinal Injuries Unit, Southern General Hospital NHS Trust, Glasgow G51 4TF, U.K.

A. J. Cathcart and S. Grant are with the Centre for Exercise Science and Medicine, Faculty of Biomedical and Life Sciences, University of Glasgow, Glasgow G12 8QQ, U.K.

S. A. Ward is with the School of Sport and Exercise Science, University of Leeds, Leeds, LS2 9JT, U.K.

Digital Object Identifier 10.1109/TNSRE.2003.819955
Conclusion: The integrated control strategy is effective in facilitating exercise testing under conditions of well-controlled cadence and power output. Our control approach significantly extends the achievable workrate range and enhances exercise-test sensitivity for FES cycling, thus allowing a more stringent characterization of physiological response profiles and estimation of key parameters of aerobic function. We further conclude that the control approach can significantly improve the overall performance of mobile recreational cycling.

Index Terms-Cardiopulmonary exercise testing, control systems, functional electrical stimulation (FES), lower limb cycling, spinal-cord injury (SCI) rehabilitation.

\section{INTRODUCTION}

\section{A. Background}

$\mathbf{L}$ OWER limb cycling by means of functional electrical stimulation (FES) of the paralyzed leg-actuating muscles of paraplegic and tetraplegic subjects has been previously described, with systems for both stationary ergometry and for mobile outdoor cycling being available [1]-[6]. A range of studies that examined the potential therapeutic and medical benefits of FES cycling exercise have recently been surveyed [7]. A key finding is that regular FES cycling exercise can lead to improved cardiopulmonary fitness, with a corresponding reduction in the likelihood of cardiovascular disease and improvements in general health. These benefits may help to reduce the general and wide-ranging effects of the secondary complications which often attend spinal-cord injury (SCI).

\section{B. Exercise Testing and Cardiopulmonary Fitness}

Most previous studies focusing on the effect of FES cycling exercise on cardiopulmonary fitness have utilized stationary FES cycling ergometers. However, with such devices, the exercise workrate and cycling cadence variables are not always well controlled. This is because, typically, the control algorithm initially attempts to maintain cadence at $50 \mathrm{r} / \mathrm{min}$ but, as fatigue develops and the stimulation level reaches its maximum value, the cadence is allowed to drop as low as $35 \mathrm{r} / \mathrm{min}$ before the resistive load is reduced in an attempt to increase cadence. Moreover, technical limitations mean that the smallest workrate increment available on these devices is limited to $6 \mathrm{~W}$, with a full workrate range typically of 0-42 W [25]. For many SCI subjects, the magnitude of this increment will be a substantial 
fraction of their maximal exercise capacity. The discriminatory power of exercise testing for cardiopulmonary and metabolic assessment is, thus, compromised, both for estimation of parameters of peak performance and also for submaximal kinetic descriptions. This is a consequence of 1) the breath-to-breath "noise" characteristic of gas exchange responses in awake human subjects, imposing a low signal-to-noise ratio [8] and 2) the limited workrate range constraining the number of discrete workrate increments that can be imposed incrementally [9], thus impairing the ability to define accurately the magnitude of any change in functional status within and between subjects.

As workrate for cycle ergometry is given by the product of angular velocity and resistive torque, the operating point of the exercise depends on both of these variables, as can the efficiency of the exercise. For example, variations in cadence at a given workrate can influence the oxygen cost of the task, reflecting the energetic cost of moving the mass of the legs [10] and the muscle fiber-type recruitment profile of the exercising muscles [11]. Thus, it is crucial that both cadence and load are well controlled. Failure to do so would represent a serious methodological weakness in FES exercise testing studies.

It is of considerable interest that Theisen et al. [12] recently proposed an FES cycling system in which cadence is regulated by feedback control of an electric motor, but stimulation intensity is kept constant, thus allowing power output to vary. One aim of the present investigation is, therefore, to develop a testbed for exercise testing in FES cycling, in which both cycling cadence and workrate are simultaneously well controlled, and in a manner that optimizes the ability to undertake accurate characterization of cardiopulmonary system response dynamics during the exercise. We achieve this using a recumbent tricycle with auxiliary electric motor, as described in the sequel.

\section{Mobile Recreational Cycling}

Several groups have described mobile FES cycles [1], [3], [4], [13]. Unfortunately, very little data reporting testing of these mobile devices with paraplegic subjects, or usage outside of laboratory conditions, have been published, and it is, therefore, difficult to assess their utility. We note that the systems described by Pons et al. [3] and Gföhler et al. [4] both included an auxiliary electric motor. In both cases, the motor is manually controlled in a feedforward manner.

In our pilot work [5], [6], we used adapted recumbent tricycles (see the following) with a low center of gravity which allows stable and independent transport without being difficult for transfer from a wheelchair. Data are reported in [6] for long-term usage by three complete-lesion paraplegic subjects, including results from outside cycling on a sports track. Thus, we have identified the potential for the recreational use of FES cycling.

A second aim of the present work is to extend the scope of mobile FES cycling by utilizing an electric motor within the overall system, and by developing an integrated strategy for feedback control of cycling cadence and leg power. There are a number of reasons for considering the addition of electric-motor assist for mobile FES cycling. A principal motivation is the relatively low power levels which can be sustained by the legs alone, exacerbated by the tendency of stimulated muscle to fatigue. In our own pilot study [6], we have found that the subjects can sustain power levels of 18-21 $\mathrm{W}$ for periods of up to $1 \mathrm{~h}$ (usually with two rest periods separating three individual cycling bouts of $20 \mathrm{~min}$ each). This performance is achieved with a relatively low-intensity training regime, consisting of only one cycling session per week. Others have reported power outputs for stationary cycle ergometry. Eser and Donaldson [14] achieved average power outputs of up to $26 \mathrm{~W}$, resulting from three weekly sessions of 30 min each. Petrofsky and Stacy [15] described more impressive results, with all of their eight subjects producing at least $55 \mathrm{~W}$ for 30 min (approximately equivalent to traveling $7 \mathrm{~km}$ ) after 24 weeks of training (however, the training frequency was not reported).

While power outputs of this magnitude are sufficient to propel a tricycle on the flat for short periods, difficulty will be experienced when faced with wind resistance and upward slopes. In addition, during the initial phases of FES cycle training, power levels are likely to be much lower than those indicated above, and may be insufficient for propulsion.

Thus, electric-motor assist is important in mobile cycling for the following reasons:

1) overall power output can be greatly increased;

2) significant disturbances can be overcome (e.g., wind resistance and slope);

3) loss of power due to muscle weakness or fatigue can be compensated for;

4) leg-cycling motion can be maintained even at very low leg power levels;

5) overall range of cycling operation can be increased.

\section{Contribution}

The integrated feedback-control strategy for FES cycling described in the sequel was designed to address the two key aims described previously: 1) to provide conditions of well-controlled cadence and workrate commensurate with the requirements of rigorous cardiopulmonary exercise testing; and 2) to significantly extend the overall performance capabilities of mobile recreational cycling.

A recumbent tricycle with auxiliary electric motor is used, which is adapted for paraplegic users and instrumented for stimulation control. The focus here is on the engineering development and testing of feedback control strategies which combine the motor power with the stimulation-induced leg power. We propose a novel integrated control strategy which simultaneously provides feedback control of leg power output (via automatic adjustment of stimulation intensity) and cycling cadence (via electric motor control). Our approach employs system identification and analytical (model-based) feedback design methods. For exercise testing, cardiopulmonary monitoring is carried out using real-time, breath-by-breath gas exchange measurements. The control strategy has been tested in experiments with both intact and complete-lesion paraplegic subjects. In this paper, we provide indicative data from one paraplegic subject. 


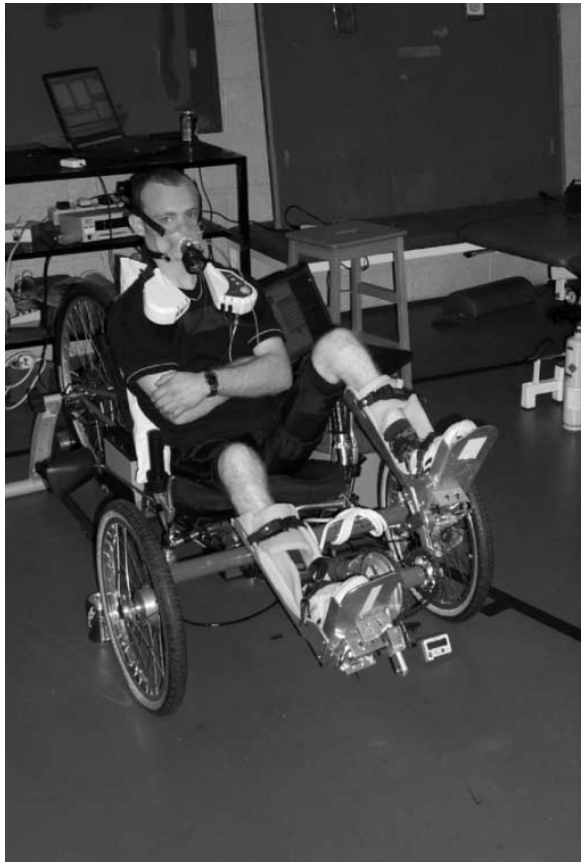

Fig. 1. Recumbent tricycle mounted on a trainer; subject connected to portable breath-by-breath gas exchange system.

\section{METHODS: ApPARATUS AND SUBJECTS}

\section{A. Apparatus}

The cycle used in this study is a standard recumbent tricycle ${ }^{1}$ which has been adapted for use by paraplegic cyclists, in conjunction with FES of the paralyzed muscles of the lower limbs (see Fig. 1). The primary mechanical alteration is the addition of customized ankle orthoses which are fixed to the pedals. These stabilize the ankle joints and constrain the legs to motion in the sagittal plane.

In addition to voluntary or FES-induced leg power, the system can be assisted by an electric motor, which is mounted behind the seat. Two battery packs are mounted on each side of the motor. The motor is directly connected through gearing to the drive wheel of the tricycle (the rear wheel), and is also directly coupled to the cranks at the front of the tricycle. Thus, even when no power is supplied by the subject, the legs can be turned by the motor. The system can be used for mobile cycling and can also be mounted on a cycle trainer for indoor exercise and testing. The cycle trainer utilized in this work ${ }^{2}$ is equipped with an electronically-controlled brake which allows different levels of resistance (load) to be set, and the total output power to be measured. The experimental results reported in the sequel were obtained using the cycle trainer setup.

\section{B. Instrumentation}

The tricycle is equipped with a 10-b shaft encoder, which is driven by a short chain attached to a cog wheel fitted to the left crank [see Fig. 2(a)]. The encoder allows measurement of the crank angle; the angular speed of the crank (i.e., the cycling

\footnotetext{
${ }^{1}$ Inspired Cycle Engineering Ltd., http://www.ice.hpv.co.uk/

${ }^{2}$ Tacx, http://www.tacx.nl/
}

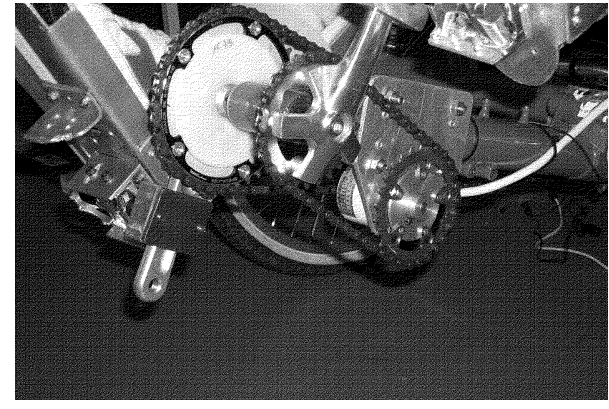

(a)

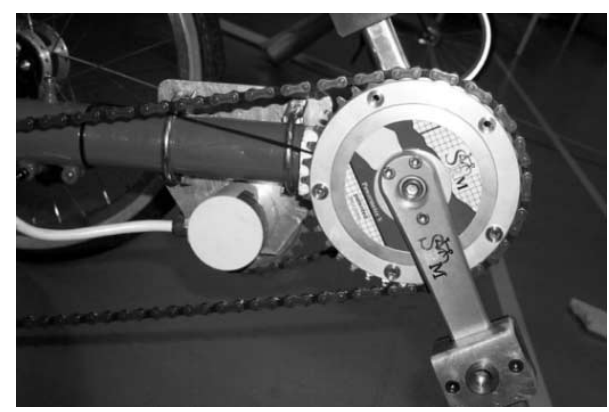

(b)

Fig. 2. Arrangement of the cranks and the torque/power and position sensors. (a) Shaft encoder and left crank arrangement, together with ankle orthoses. (b) Right crank with torque/power measurement sensor.

cadence) is obtained by differentiation (with filtering). The tricycle has a throttle fitted to the left hand grip. The throttle can be used by the cyclist in a number of ways, depending on the control software configuration: it can be used to directly control the motor input signal; it can alternatively be used in FES cycling to directly vary the stimulation intensity; or in closed-loop control configurations it can be used to set the reference values for the controlled variables (e.g., desired cadence or leg power). The right crank of the tricycle is replaced by a torque measurement sensor $^{3}$ [resolution $\pm 2.5 \%$, see Fig. 2(b)]. The sensor operates on the basis of four strain gauges and this allows the torque produced at the cranks to be measured. The sensor also provides a measurement of cycling cadence. Thus, instantaneous leg power input can be computed as the product of instantaneous torque and angular speed.

The signals measured from the throttle, shaft encoder, torque sensor, and the motor are interfaced to a data acquisition card installed in a laptop computer. These signals are processed by the control software running in the laptop in order to produce control signals for the stimulation intensity (as described in the following section) and for the motor. The realtime control software is implemented in Matlab/Simulink, ${ }^{4}$ in conjunction with the Real-Time Toolbox. The sampling frequency for feedback control was $20 \mathrm{~Hz}$ (i.e., sample interval of $50 \mathrm{~ms}$ ), which corresponds to the muscle stimulation frequency (see the following).

\section{Stimulation Patterns}

The stimulation control software running in the laptop controls a multichannel stimulator (the stimulator is described in [16]).

${ }^{3}$ Schoberer Rad Messtechnik (SRM), http://www.srm.de/

${ }^{4}$ The MathWorks Inc., http://www.mathworks.com/ 


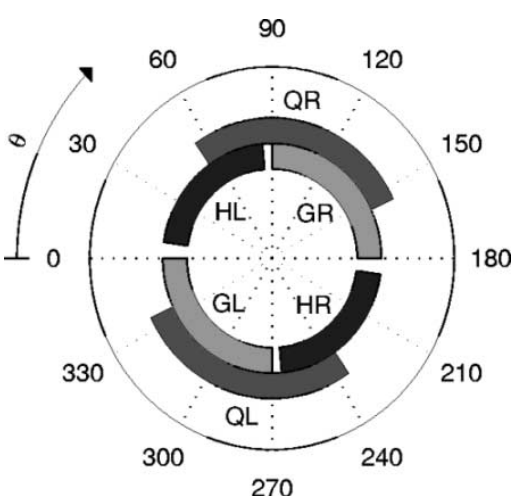

(a)

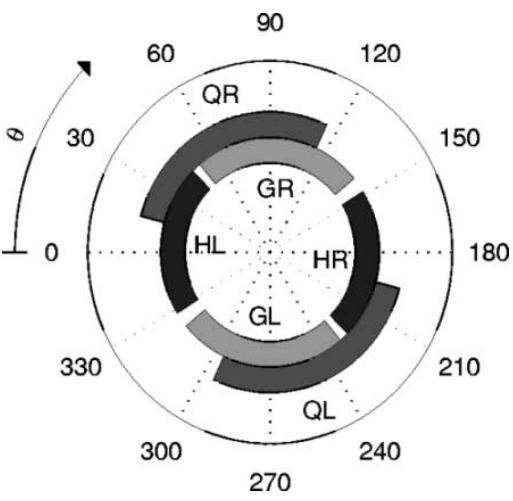

(b)

Fig. 3. Stimulation patterns: QL-quadriceps left; QR-quadriceps right; $\mathrm{HL}$-hamstrings left; HR — hamstrings right; GL—gluteus left; GR — gluteus right. (a) Static stimulation pattern. (b) Stimulation pattern at $50 \mathrm{r} / \mathrm{min}$.

During the experiments reported here, the stimulator operated at constant frequency $(20 \mathrm{~Hz})$. The stimulation current is adjustable in $10 \mathrm{~mA}$ increments up to a maximum of 120 $\mathrm{mA}$. The current for each channel of stimulation was individually adjusted at the start of each cycling session, and then fixed for the remainder of the session. The stimulation intensity can be varied during cycling by adjustment of the pulsewidth across a range of $0-800 \mu$ s. The same pulsewidth was applied to each channel.

Surface electrodes were attached to six muscle groups, i.e., the left and right quadriceps, hamstring, and gluteal muscles. The crank angle measurement was used to switch each muscle group on and off during each cycle according to a prespecified pattern. Typical stimulation patterns are shown schematically in Fig. 3, where the crank angle is denoted as $\theta$. The arcs in the diagrams show the angle ranges where each muscle group is stimulated. The "static" stimulation patterns shown in Fig. 3(a) were obtained individually for each muscle group under static conditions (i.e., at fixed points on the cycle) by manual determination of the positions where significant positive torque was produced by stimulation of the corresponding muscle group. In order to compensate for the dynamic response of the muscle (i.e., the time taken for maximal force to be produced following onset of stimulation), the stimulation patterns have to be shifted forward in time as cycle cadence increases. This is illustrated in Fig. 3(b), where the patterns for a cadence of $50 \mathrm{r} / \mathrm{min}$ are shown. The shift angle is proportional to cycling cadence.

\section{Gas Exchange Monitoring}

Using a portable breath-by-breath system, ${ }^{5}$ and with the subject breathing through a low deadspace mask, respired $\mathrm{O}_{2}$ and $\mathrm{CO}_{2}$ concentrations and respired volume and flow were monitored continuously by discrete gas analyzers and a turbine, respectively (see Fig. 1). Oxygen uptake $\left(\dot{\mathrm{VO}}_{2}\right)$ and related gas exchange variables were calculated in real-time on a breath-bybreath basis [17]. $\dot{\mathrm{VO}}{ }_{2}$ responses for each exercise test were edited to remove outliers, and then timeaveraged for subsequent analysis.

\section{E. Subjects}

The control strategies described in the sequel have been tested in a series of experiments with both intact and paraplegic subjects. Physiological response data are presented for one male paraplegic subject with a motor-complete spinal cord lesion at level T8/9. He had previously been participating in a pilot study of FES cycling for approximately 18 months, with one 1-h cycling session per week (details of the pilot study are given in Hunt et al. [6]). He was three years post-injury at the start of the pilot study, at which time he was aged 28 years. All procedures were approved by the Southern General Hospital Ethics Committee; the subject provided informed consent prior to participation.

\section{Methods: FeEdBACK Control Structures AND DESIGN APPROACH}

\section{A. Integrated Control Strategy}

The motorized and instrumented tricycle can be used for simultaneous feedback control of cycling cadence and of leg power output, combined with manual control of total power output at the drive wheel (by adjusting the drive wheel resistance using the electronic brake). We propose an integrated control scheme with two independent feedback loops, as shown in Fig. 4. In the first loop, the electric motor input is automatically adjusted in such a way that the cycling cadence (or, equivalently, depending on the gear engaged, the cycle's forward speed) is controlled to a reference value by feedback. This feedback loop has a relatively high bandwidth, and is designed to compensate for other disturbing influences which affect the cadence, including muscle weakness and fatigue, wind resistance, friction, and slope.

The second loop provides feedback control of leg power, as measured at the cranks. Stimulation intensity (here, pulsewidth) is automatically adjusted to keep the measured power close to a reference value, which can be set arbitrarily in the control software.

The net effect of this control scheme is that smooth cycling motion at constant cadence can always be achieved by the motor control loop, even if the leg power contribution varies or becomes low as a result of fatigue, or if the total load changes. Effectively, the total mechanical power output of the rider-tricycle system, which is comprised of the sum of motor power and leg

\footnotetext{
${ }^{5}$ MetaMax 3B, Cortex Biophysik GmbH, Germany.
} 


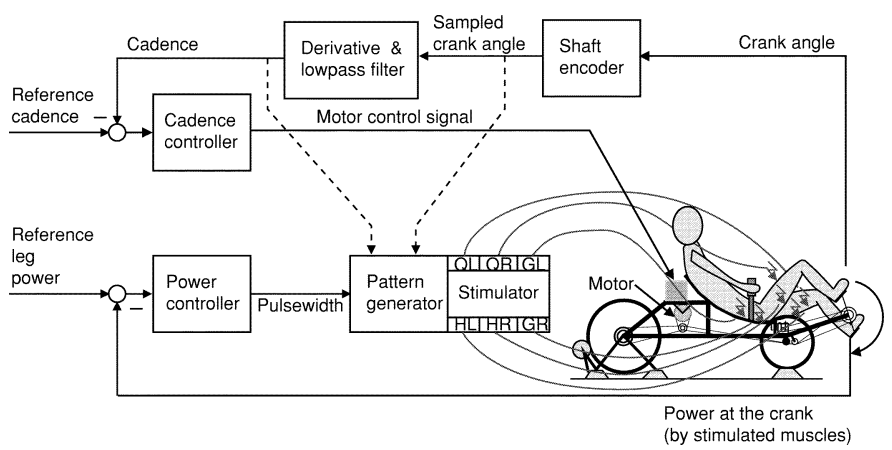

Fig. 4. Integrated closed-loop control scheme. One loop automatically adjusts the motor input to keep the cycling cadence close to a reference value set by the throttle. The second loop automatically adjusts the stimulation pulsewidth to keep the leg power close to an arbitrary reference value.

power, is automatically varied in order to maintain the instantaneous value of desired cadence. Independently, the muscle stimulation loop attempts to keep the muscles working at an arbitrarily specified workrate, and, thus, it is the motor power which automatically varies in order to meet the overall total instantaneous power requirements.

Thus, the leg power output, which represents the subject's workrate, can be well-controlled to arbitrary values ranging from zero-stimulation workrate (which is negative-see later in the paper) up to the level obtained with maximal stimulation intensity. The level of the desired leg power can, thus, be chosen to keep the subject's legs working at an "optimal" operating condition, or to achieve a prespecified workrate profile for exercise testing (e.g., step or incremental).

\section{B. System Identification and Feedback Design}

For the two control loops, feedback control design is based upon a two-stage procedure. First, the open-loop dynamics are identified empirically by applying a test input signal and measuring the resulting output. The input-output dynamics are then estimated as linear transfer functions using a linear least squares approach [18]. For the motor control loop, the open-loop input is the motor input signal, and the output is the cycling cadence (crank angular speed). For the stimulation loop, the open-loop input is the stimulation pulsewidth, and the output is the resulting leg power measured at the crank.

In the second stage for each loop, the identified transfer function is used within an analytical (model-based) control design procedure which delivers the linear transfer function of the corresponding controller. Here, we have employed the pole assignment design procedure [19]. This allows specification of the nominal (desired) closed-loop risetime and damping for each loop.

The experimental procedure for identification and control design for each loop consists of the following steps.

1) Motor Loop: The subject sits normally on the tricycle with legs attached to the pedal orthoses. No stimulation is applied in this step.

a) Open-loop pseudo-random binary sequence (PRBS) test: A motor input signal of PRBS (see
[18]) form is applied in open loop. The PRBS signal has a specified mean level (usually close to the midrange of the input signal) and amplitude. The resulting cadence is measured.

b) Model identification: A linear dynamic (transfer function) model is fit to the input-output (motor signal-cadence) data using a least squares criterion. A range of model orders can be tested and models compared and validated using a separate data set.

c) Control design: The validated model is used to design the feedback controller for cadence. Pole assignment design is employed, with specified closed-loop risetime and damping.

2) Stimulation Loop: This step is carried out with the subject on the tricycle, and with the cycle-cadence controller designed in the previous step operational and controlling the cadence at a constant reference value (typically $50 \mathrm{r} / \mathrm{min}$ ). Under these conditions, the following steps are carried out:

a) Open-loop PRBS test: A muscle stimulation pulsewidth signal of PRBS form is applied in open loop. The resulting leg power is measured at the crank.

b) Model identification: A linear dynamic (transfer function) model is fit to the input-output (pulsewidth-leg power) data and validation is carried out.

c) Control design: The identified model is used to design a feedback controller for leg power. Pole assignment design is also employed for this loop, with specified closedloop risetime and damping.

Matlab/Simulink has been used for both system identification (the System Identification Toolbox ${ }^{6}$ ) and control design (the Polynomial Toolbox ${ }^{7}$ ).

\section{Closed-Loop Frequency Response}

To permit analysis of the frequency-domain properties of the feedback loops, consider the generic loop structure shown in Fig. 5. Here, the plant to be controlled is represented by the frequency-dependent transfer function $P$, while the corresponding dynamic controller is $C . u$ is the plant input and $y$ the output to be controlled to follow the reference signal $r$. The net effect of disturbances is represented by the signal $d$, while $n$ is a measurement noise. The generic structure is valid for both feedback loops considered in the motor-assisted cycling problem. For example, for the stimulation loop, $P$ is the stimulated musculo-skeletal system of the legs, $u$ is the stimulation pulsewidth, $y$ is the resulting leg power, and $r$ is the desired reference value for leg power. $d$ represents the net effect of disturbances affecting the leg power output.

The closed-loop equation for the generic loop is

$$
y=\frac{C P}{1+C P} r+\frac{1}{1+C P} d-\frac{C P}{1+C P} n .
$$

\footnotetext{
${ }^{6}$ The Mathworks Inc., http://www.mathworks.com/
}

${ }^{7}$ Polyx Ltd., http://www.polyx.com/ 


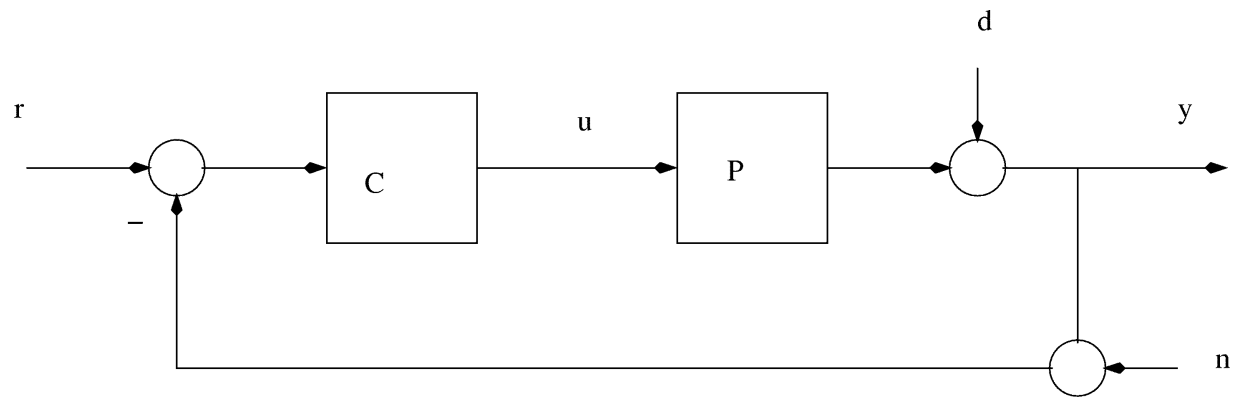

Fig. 5. Generic feedback loop.

Defining the sensitivity function as $S=1 /(1+C P)$ and the complementary sensitivity function as $T=C P /(1+C P)$, the closed-loop equation becomes

$$
y=T r+S d-T n .
$$

Thus, $S$ determines the disturbance rejection properties of the loop, while $T$ defines the reference tracking response and the effect of measurement noise.

\section{Feedback Control Tests}

In order to test the efficacy of the integrated feedback-control strategy, three separate feedback-control tests have been defined. During all of these tests, both of the feedback loops are operational.

1) Test CT: Cadence tracking control. This is a closed-loop test of cycle cadence tracking. During this test, the legpower reference value is kept constant, but the reference cadence is subject to step changes.

2) Test PT: Power tracking control. This is a closed-loop test of tracking of leg power output. The reference signal for the cycle cadence control loop stays constant, but the leg power reference changes in steps.

3) Test DR: Disturbance rejection. During this test, the reference values for cycle cadence and leg power are both kept constant. The resistance acting at the drive wheel is then varied in order to simulate changes in load and, therefore, changes in the required total mechanical power output.

The total mechanical output power is measured at the drive wheel during each of these tests.

\section{E. Exercise Testing}

We have carried out two types of standard exercise test (step and incremental), during which online monitoring of pulmonary gas exchange is performed on a breath-by-breath basis. To impose the desired workrate forcing function effectively, accurate feedback control of both cadence and leg power is crucial.

1) Submaximal step test. Here, feedback control of cycle cadence is operational, with a constant cadence setpoint throughout. At the start of the test, the legs are turned by the electric motor, while the muscle stimulation is zero. Subsequently, the leg power reference (i.e., exercise workrate) is set to $0 \mathrm{~W}$ (with stimulation enabled, and being automatically adjusted by feedback) for a time sufficient to allow the gas exchange responses to stabilize. The leg power reference is then set to a constant, submaximal level for the remainder of the test.

2) Incremental test. Again, constant-cadence control is operational during the whole test. In this type of test, the legs are initially turned by the electric motor, while the muscle stimulation is zero, for sufficient time to allow the gas exchange responses to stabilise at a new steady state. Then, with stimulation enabled and under feedback control, the leg power reference is incremented by a small, arbitrary amount and held constant at this level for some pre-specified time (i.e., $1 \mathrm{~min}$ ) before the next increment is imposed. These periodic workrate increments are continued until the subject's maximal workrate is reached [9].

\section{EXPERIMENTAL RESULTS}

\section{A. System Identification}

The open-loop identification data and the output of the identified model for the motor-cadence system are shown in Fig. 6. The total data set is shown in Fig. 6(a), while the zoomed plot of Fig. 6(b) focuses on part of the data used for model validation, thus allowing the model-simulated output to be compared with the measured output. The normalized motor input signal has a PRBS form, and is shown in the lower part of the graphs. The measured cycle cadence, induced by the PRBS input, is the solid line in the upper part of the graphs. The high-frequency ripple in the measured output is caused by the weight of the legs during each cycle (the frequency of the ripple is the same as the cycling cadence). These input-output data were used to identify linear models, and it was found that a second-order linear transfer function was sufficient for good approximation. The second-order model was simulated with the PRBS input which was applied to the physical system, using a short sequence from the overall data set - the simulated output is plotted as the dashed line in the upper graphs of Fig. 6 [best seen in the zoomed plot of Fig. 6(b)]. It is seen that the model captures the dominant dynamic response of the motor loop. For the purposes of control design, it is unnecessary for the model to capture the high-frequency gravitational effects of the legs acting on the pedals, since it is not desirable for the controller to try and counteract these effects. The identified model was used to compute a feedback controller for cycle cadence, as described previously.

Identification results for the stimulation-leg power system are shown in Fig. 7. Here, the input signal (pulsewidth) has a 

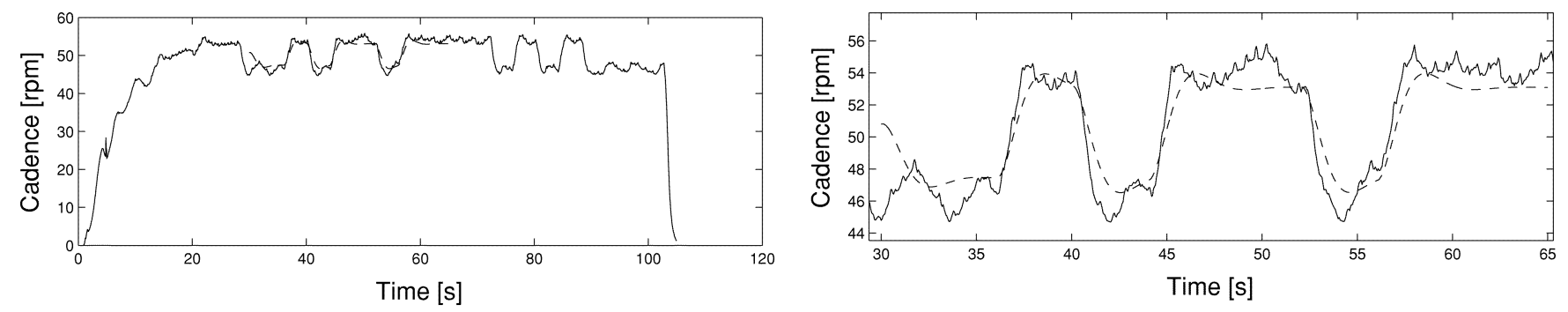

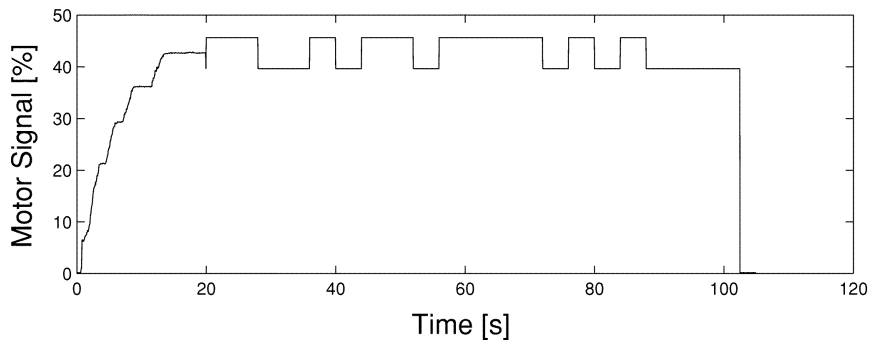

(a)

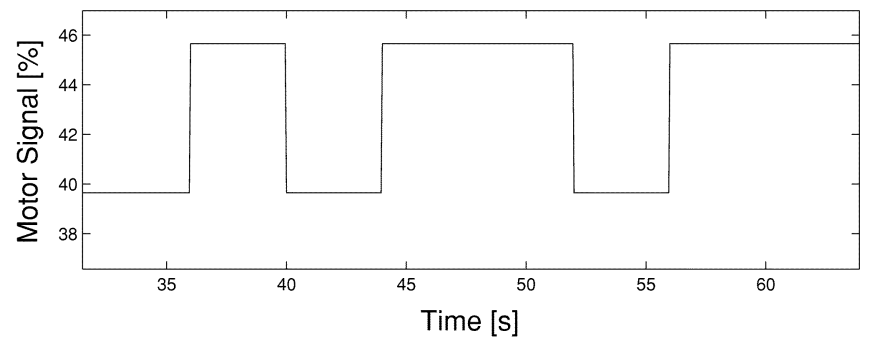

(b)

Fig. 6. Identification of motor input-cadence system. The normalized motor input signal is shown in the lower graphs. The upper graphs show the corresponding cycle cadence (solid line). The dashed line in the upper graphs is the output of the identified model. (a) Total data set. (b) Zoom into part of data set used to illustrate and compare simulated model output with measured signal (validation).


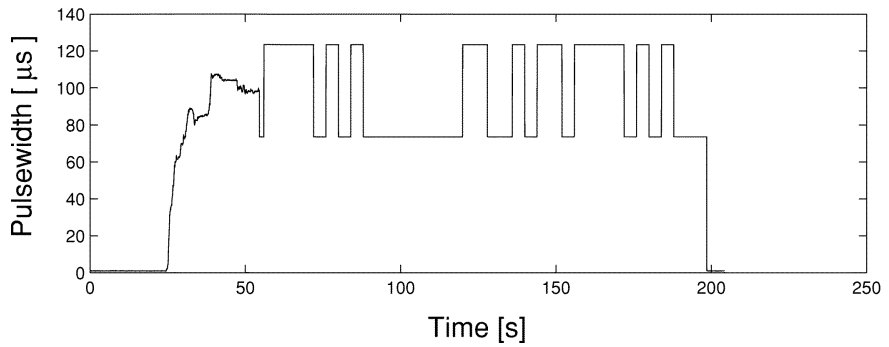

(a)

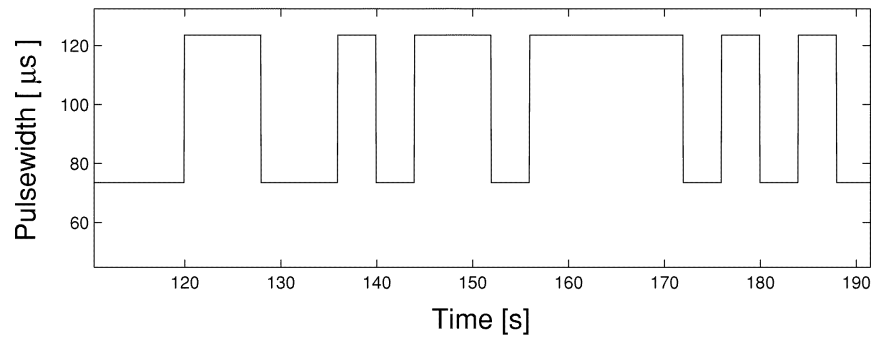

(b)

Fig. 7. Identification of stimulation-leg power system. The pulsewidth input signal is shown in the lower graphs (PRBS signal). The upper graphs show the corresponding leg power (solid line). The dashed line in the upper graphs is the output of the identified model. (a) Total data set. (b) Zoom into part of data set used to illustrate and compare simulated model output with measured signal (validation).

PRBS form and is shown in the lower part of the graphs. The corresponding measured leg power output is shown as the solid line in the upper part of the figure. During the test, the cadence was automatically controlled to $50 \mathrm{r} / \mathrm{min}$. A first-order model was found to be sufficiently accurate. The simulated model output, corresponding to the PRBS input signal, is the dashed line in the upper plots of Fig. 7. Again, the high-frequency ripple at 50 $\mathrm{r} / \mathrm{min}$ is not relevant for modeling and control design. The model was used to design a feedback controller for leg power.

\section{B. Control Design}

The key closed-loop transfer functions for the motor loop and the stimulation loop are shown in Fig. 8. The plots of $|S|$ and $|T|$ show that the gravitational leg ripple has a frequency well outside the bandwidth of both loops [it is $(50 \mathrm{r} / \mathrm{min}) /(60 \mathrm{~s}$. $\left.\min ^{-1}\right)=0.833 \mathrm{~Hz}$, or $\left.5.2 \mathrm{rad} / \mathrm{s}\right]$. This means that the ripple will not affect the control signal, i.e., that neither controller attempts to attenuate this disturbance (since $|S| \approx 1$ at this frequency for both controllers).

\section{Feedback Control Tests}

The results of feedback control tests CT, PT, and DR are shown in Figs. 9-11. The convention adopted in these figures is that all of the upper plots show the controlled variable (i.e., cadence or leg power), plotted as a solid line. Also shown in each of the upper plots is the reference signal for the corresponding variable, which is plotted as a dotted line. Since the feedback control target is always to make the controlled variable follow the reference, it frequently happens that the reference signal plot is obscured by the controlled variable, particu- 


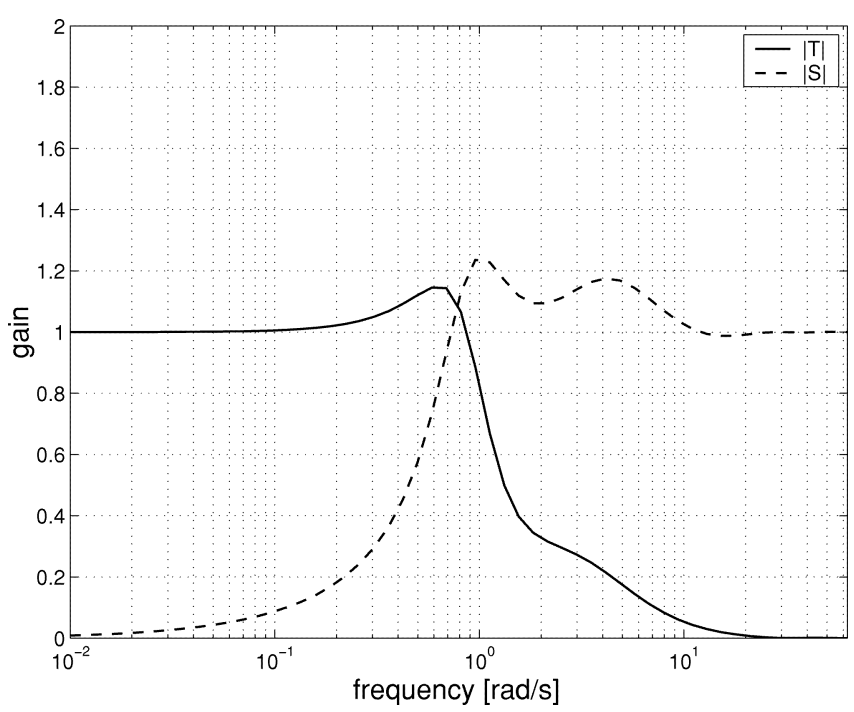

(a)

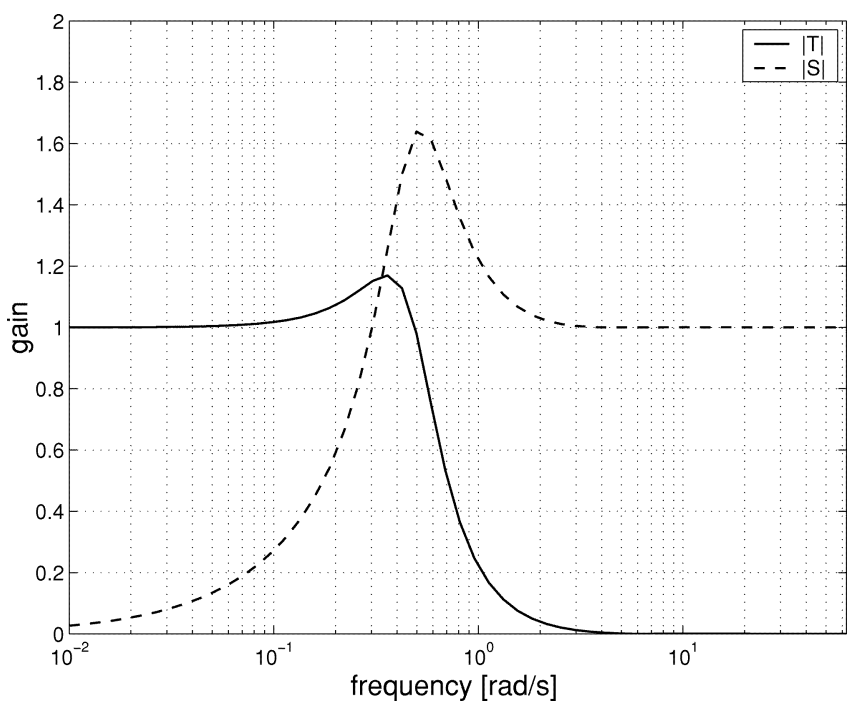

(b)

Fig. 8. Magnitude of closed loop sensitivity functions (see text). (a) Motor loop. (b) Stimulation loop.

larly during periods where the reference is held constant for a time. The upper graphs also contain the "ideal response" of the controlled variable, i.e., the response achieved when the given controller is simulated with the nominal plant model. The ideal response is plotted as a dashed line, which again is sometimes obscured by the controlled variable line.

The lower plots in Figs. 9-11 all show the appropriate control input for the given test (i.e., for cadence control it is the motor input signal, while for leg power control it is the stimulation pulsewidth).

Cadence Tracking Control (Test CT): The results of Test CT are shown in Fig. 9. During this test, the cadence reference is changed in a step-wise fashion, while the reference signal for the leg power is kept constant. The controlled cadence (solid line) and reference signal (dotted line) are shown in the upper part of Fig. 9(a), and the corresponding motor input signal can be seen in the lower plot. The dashed line in the upper plot is the ideal cadence response, i.e., the response achieved when the cadence controller is simulated with the nominal plant model. It can be seen that accurate and fast cadence tracking is achieved by the feedback controller. As discussed previously, the highfrequency cadence ripple is not attenuated by the controller, and has only a small effect on the motor input.

The corresponding response of the stimulation-leg power loop during Test CT is shown in Fig. 9(b). The reference signal for leg power is constant at $10 \mathrm{~W}$. The upper part of Fig. 9(b) shows the controlled leg power (solid line), the reference power (dotted line, 10-W constant), and the ideal controlled power response (dashed line). During the first $10 \mathrm{~s}$ only of this test the legs were assisted manually by an experimenter, resulting in the large initial value of leg power $(>20 \mathrm{~W})$ seen in the plot. The corresponding stimulation pulsewidth is plotted in the lower part of Fig. 9(b). It can be seen that, following the initial transient, the power controller keeps the leg power close to the desired value, despite the disturbances which are introduced as a result of changes in the cycling cadence. The stimulation pulsewidth is seen to be smooth throughout.

Power Tracking Control (Test PT): The results of Test PT are shown in Fig. 10. During this test the reference signal for leg power is changed in steps, while the cadence reference is constant. The upper plot in Fig. 10(b) shows the reference leg power (dotted line) and the measured leg power (solid line), together with the ideal (simulated) power response (dashed line, obtained using the controller and identified model). As in Test $\mathrm{CT}$, the experimenter manually applied a positive torque to the legs during the first few seconds of the test, resulting in the large initial value of leg power $(>20 \mathrm{~W})$ seen in the plot. The corresponding stimulation pulsewidth is shown in the lower part of the figure.

The response of the motor signal-cadence control loop during this test is shown in Fig. 10(a). The upper part of the figure shows the reference cadence (dotted line, constant $50 \mathrm{r} / \mathrm{min}$ ), the measured cadence (solid line), and the ideal cadence response (dashed line). The corresponding motor input signal is shown in the lower part of Fig. 10(a).

Disturbance Rejection (Test DR): The results of a disturbance rejection test are shown in Fig. 11. During the test, the reference values for both the cadence and for the leg power are kept constant. The resistance on the cycle trainer is then repeatedly varied between its minimum and maximum values. Since the cycling cadence is kept approximately constant during this test, the effect of varying the resistance is to vary the total power output at the drive wheel: minimum resistance corresponds in this test to a total power of $15 \mathrm{~W}$, while maximum resistance resulted in a total output power of $49 \mathrm{~W}$.

The controlled cadence (solid line) and reference signal (dotted line) are shown in the upper part of Fig. 11(a), and the corresponding motor input signal can be seen in the lower plot. The dashed line in the upper plot is the ideal cadence response. The response of the stimulation-leg power loop during Test DR is shown in Fig. 11(b). The upper part of Fig. 11(b) shows the controlled leg power (solid line), the reference power (dotted line), and the ideal controlled power response (dashed line). The corresponding stimulation pulsewidth is plotted in the lower part of Fig. 11(b).

It can be seen that the motor input, which is continually adjusted by feedback, undergoes larger changes every $20 \mathrm{~s}$ as the 

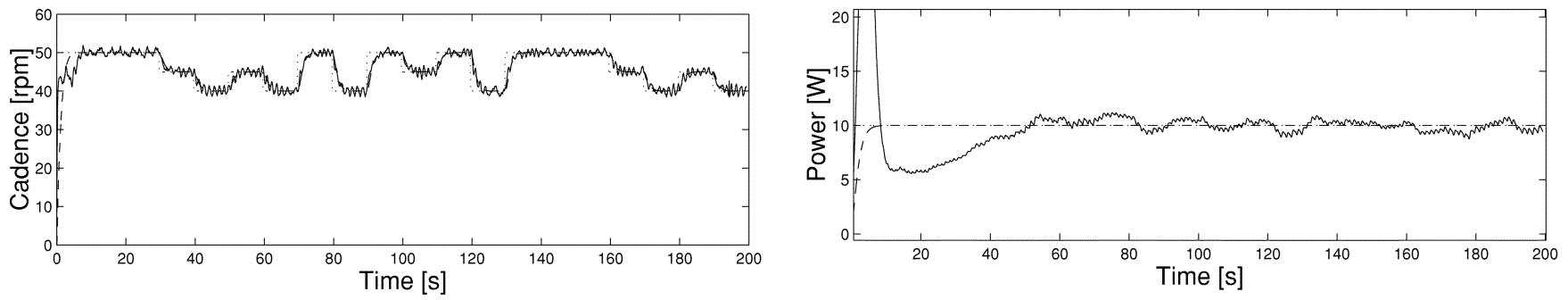

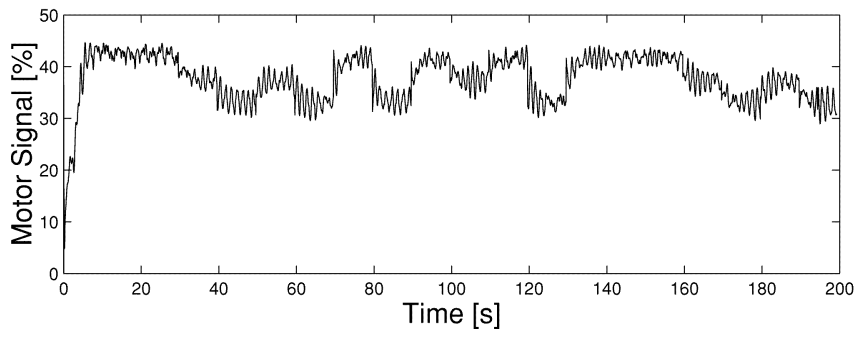

(a)

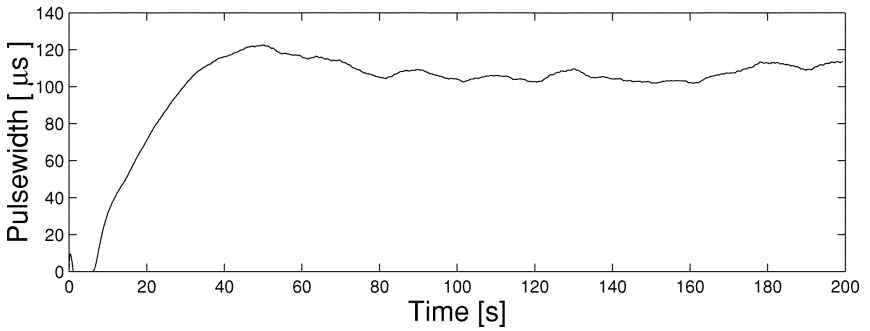

(b)

Fig. 9. Test CT. (a) Motor loop response. The upper graph shows the reference cadence (dotted line), the measured cadence (solid line), and the ideal cadence response (dashed line). The lower plot shows the motor input signal. (b) Stimulation loop response. The upper graph shows the controlled leg power (solid line), and the ideal power response (dashed line). The reference leg power is constant at $10 \mathrm{~W}$ (dotted line). The lower plot shows the stimulation pulsewidth.
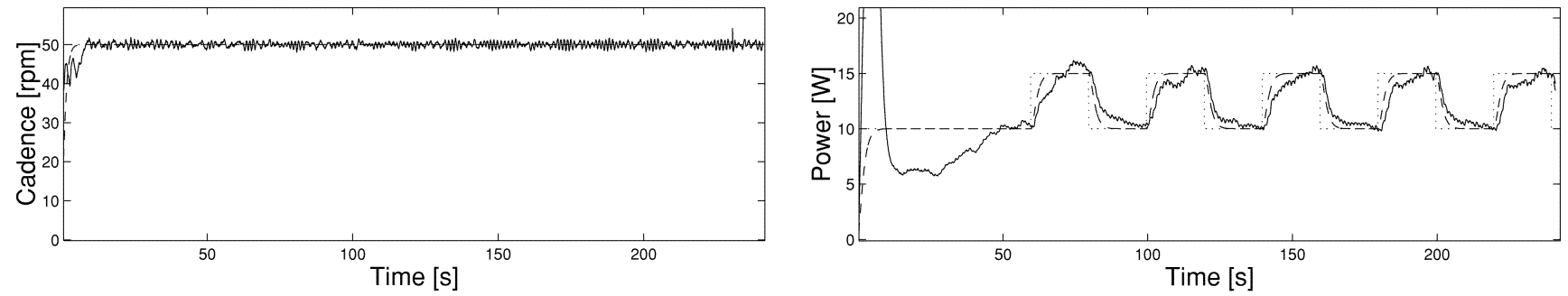

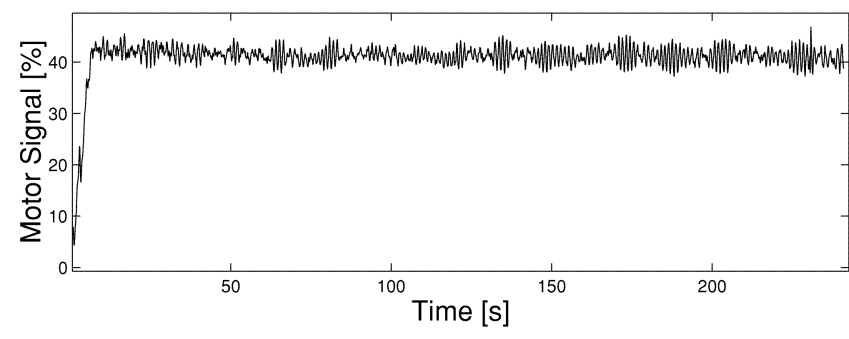

(a)

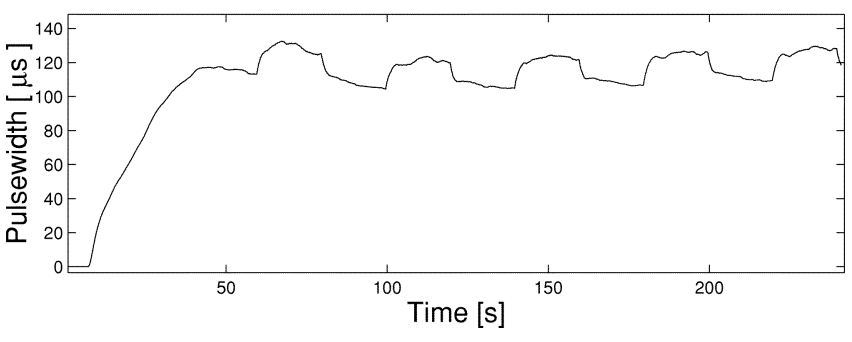

(b)

Fig. 10. Test PT. (a) Motor loop response. The upper graph shows the reference cadence (dotted line, constant at $50 \mathrm{r} / \mathrm{min}$ ), the measured cadence (solid line), and the ideal cadence response (dashed line). The lower plot shows the motor input signal. (b) Stimulation loop response. The upper graph shows the reference signal for leg power (dotted line), the controlled leg power (solid line), and the ideal power response (dashed line). The lower plot shows the stimulation pulsewidth.

load (trainer resistance) is changed between its minimum and maximum values. Despite these load changes, both cadence and leg power output are maintained close to their desired, constant values.

\section{Exercise Tests With Pulmonary Gas Exchange Monitoring}

1) Submaximal Step Test: The results of a submaximal step exercise test, with simultaneous feedback control of cycling cadence and leg power, are shown in Fig. 12. This test was carried out at a controlled cadence of $50 \mathrm{r} / \mathrm{min}$, as indicated in the top graph in the figure.

During the first 4 min of the test, the legs were turned at this cadence, but without stimulation (zero pulsewidth). This was achieved by setting the power reference to a value lower than the "retarding torque" observed without stimulation. For the next 6 min (240-600 s), the leg power reference was set to zero (see the "power" plot in the upper graph-here, both reference power [dotted line] and measured power [solid line] are shown together, and labeled "power"-for $t>240 \mathrm{~s}$ the feedback control is sufficiently accurate that these two lines coincide). The average stimulation pulsewidth during this phase was just over $100 \mu \mathrm{s}$, as shown in the middle graph of Fig. 12. Stimulation is necessary even for zero leg power output, since energy input is required to overcome the inertial moment of the legs. At $t=600 \mathrm{~s}$, the leg power reference was then set to the submaximal level of $12 \mathrm{~W}$. As seen in the upper graph, this power output was achieved quickly and then accurately maintained by feedback for the remainder of the test. It is noted that, in order to maintain the constant workrate of $12 \mathrm{~W}$, the feedback control requires to continuously increase the stimulation pulsewidth (middle graph). This is a consequence of muscle fatigue. 

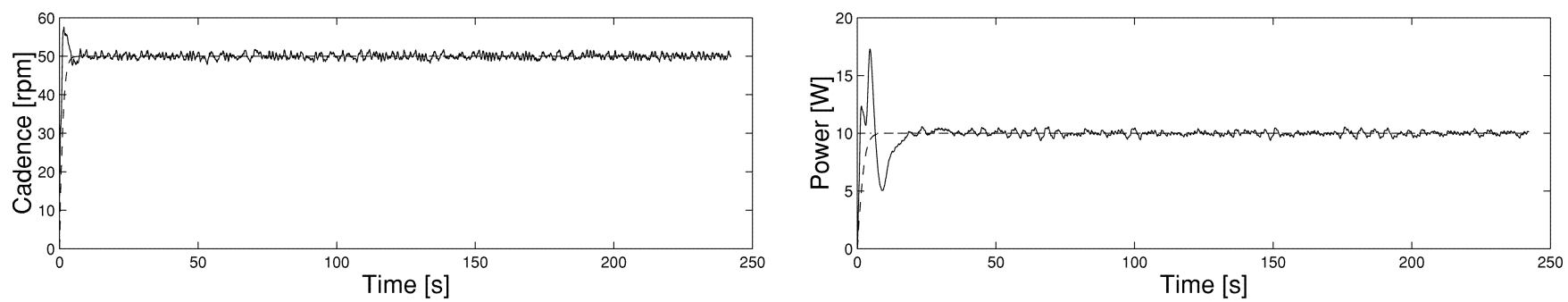

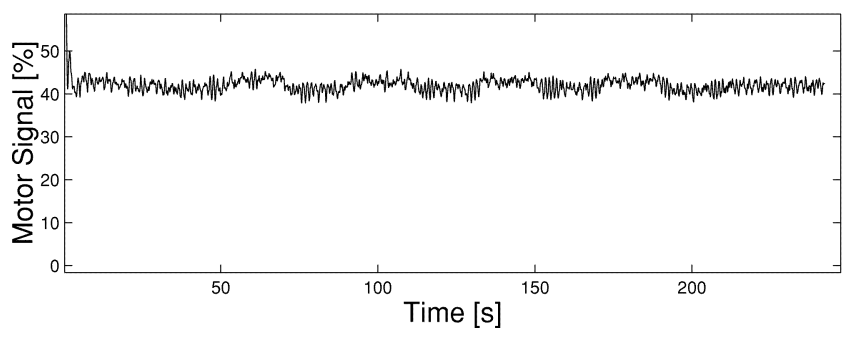

(a)



(b)

Fig. 11. Test DR. (a) Motor loop response. The upper graph shows the reference cadence (dotted line, constant at 50 rpm), the measured cadence (solid line), and the ideal cadence response (dashed line). The lower plot shows the motor input signal; (b) Stimulation loop response. The upper graph shows the reference signal for leg power (dotted line), the controlled leg power (solid line), and the ideal power response (dashed line). The lower plot shows the stimulation pulsewidth.
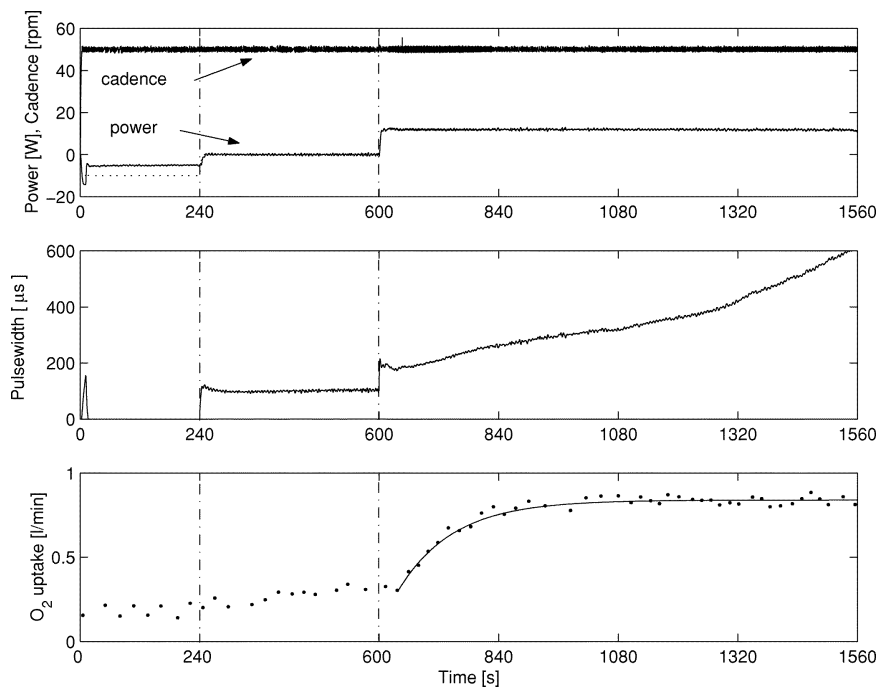

Fig. 12. Responses to submaximal step exercise test. Solid line in lower graph is best-fit exponential $\dot{\mathrm{VO}}_{2}$ "phase II" response.

The corresponding oxygen uptake response $\left(\dot{\mathrm{V}}_{2}\right.$, in $1 \cdot \min ^{-1}$ ) is shown in the lower graph in Fig. 12. The $\dot{\mathrm{V}}_{2}$ response was well described by an exponential function, constrained to fit the predominant "phase II" component of the response (i.e., by excluding the initial 15-20 s following the workrate increase when gas exchange is determined by any associated increase in cardiac output [20])

$$
\dot{\mathrm{V}} \mathrm{O}_{2}(t)=\dot{\mathrm{V}} \mathrm{O}_{2}(0)+\Delta \dot{\mathrm{V}} \mathrm{O}_{2}^{\mathrm{ss}}\left(1-e^{\frac{-(t-\delta)}{\tau}}\right)
$$

where $\Delta \dot{\mathrm{V}} \mathrm{O}_{2}^{\text {ss }}$ is the magnitude of the steady-state increase in $\dot{\mathrm{V}} \mathrm{O}_{2}$ resulting from the step increase in workrate, $\tau$ is the time constant and $\delta$ is a delay term reflective of (but not equal to) the phase I-phase II transition time. $\Delta \dot{\mathrm{V}} \mathrm{O}_{2}^{\mathrm{ss}}$ was $530 \mathrm{ml} \cdot \mathrm{min}^{-1}$, while $\tau$ was $110 \mathrm{~s}$.

2) Incremental Exercise Test: Incremental exercise test results are shown in Fig. 13. Simultaneous feedback control
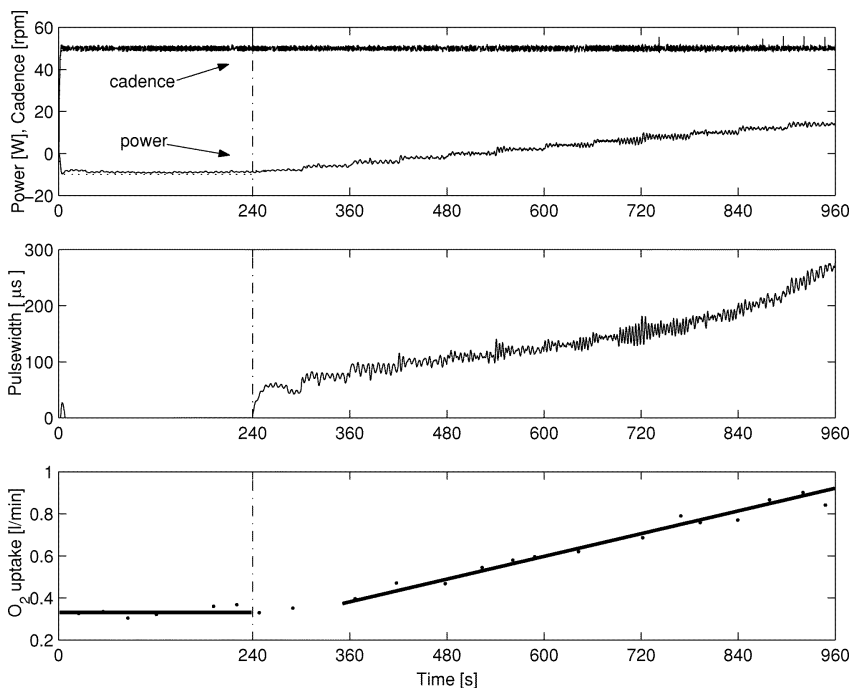

Fig. 13. Responses to a maximum incremental exercise test. Solid line in lower graph is the best-fit to the linear phase of the $\mathrm{V}_{2}$ response.

of cycling cadence and leg power was utilized, at a controlled cadence of $50 \mathrm{r} / \mathrm{min}$ (Fig. 13, upper graph). During the first $4 \mathrm{~min}$, the legs were turned at this cadence, but without stimulation. This was achieved by setting the power reference to a value lower than the "retarding torque" observed without stimulation. Subsequently, the power reference was increased in steps of $2 \mathrm{~W}$ each minute. Note that the "power" plot in Fig. 13 shows both the reference power (dotted line) and the measured leg power (solid line)- the power controller is sufficiently accurate that these two signals are indistinguishable, for $t>240 \mathrm{~s}$. Power increments were increased until the stimulation pulsewidth reached a prespecified limit of $600 \mu \mathrm{s}$ (Fig. 13, middle graph, truncated at $t=960 \mathrm{~s}$ ), at which point no further increase in power output is possible, and the test was, therefore, discontinued.

Following the imposition of the stimulation at $240 \mathrm{~s}, \dot{\mathrm{V}}_{2}$ (in $1 \cdot \mathrm{min}^{-1}$, Fig. 13, lower graph) increased linearly with time and 
therefore workrate, after an initial "kinetic" phase that reflects both the vascular transit delay between the exercising limbs and the lungs and the system response kinetics; i.e. the laggedlinear behavior expected of a first-order system [21]. This response resembles those described for volitional ramp cycle ergometry in healthy subjects [21], but with a substantially steeper ramp slope $\left(\Delta \dot{\mathrm{VO}}_{2} / \Delta\right.$ workrate $)$ of $23.8 \mathrm{ml} \cdot \mathrm{min}^{-1} \cdot \mathrm{W}^{-1}$. We elected not to report a mean response time, however, as its computation requires knowledge of the corresponding steady-state $\dot{\mathrm{V}} \mathrm{O}_{2}$-workrate relationship [21] which, at this point, we have not yet formally defined.

\section{DisCUSSION OF RESUlTS}

The results of this investigation demonstrate that accurate feedback control of cycling cadence and exercise workrate can be achieved simultaneously. Both loops give good responses to changes in their reference values, and both loops are able to maintain their reference values while significant load changes take place. Results of feedback control of workrate during exercise tests show that the subject's leg power output (workrate) can be controlled to an arbitrary reference value, and workrate increments can be arbitrarily small. Thus, our control approach significantly extends the functional workrate range for FES cycling exercise, because the exercise baseline is the workrate corresponding to zero stimulation input (in the tests shown, the workrate baseline is approximately $-9 \mathrm{~W}$ ), rather than a $0-\mathrm{W}$ baseline (which requires a stimulation input sufficient to fully rotate the mass of the legs). In our approach, the stimulation can be gradually increased from $0 \mu$ s such that the exercise workrate begins at around $-9 \mathrm{~W}$ and then increases gradually toward 0 W and beyond. During the "negative workrate" phase, the subject's legs are not contributing to the total mechanical work done against the load but, as stimulation increases the workrate toward $0 \mathrm{~W}$, the muscles perform an increasing amount of work to move the legs, thus decreasing the level of work done by the electric motor to move the legs. When the workrate rises above $0 \mathrm{~W}$, the legs begin to contribute to the total work done against the load.

It was of interest that (in this subject, at least) the corresponding $\dot{\mathrm{V}}_{2}$ response kinetics to both the step and the incremental FES forcings appeared to be well described as linear and first-order (i.e., meeting the criterion of superposition). This is consistent with earlier descriptions for volitional cycle ergometry in healthy subjects [22]. However, the $\dot{\mathrm{V}}_{2}$ kinetics were substantially slower than normal, consistent with the poor conditioning and muscle atrophy typical of the SCI population [23], [24]. In addition, the high $\mathrm{O}_{2}$ cost of both the step and incremental exercise is consistent with earlier reports in SCI subjects undergoing FES (e.g., [25]), being over two-fold higher than reported for volitional cycle ergometry [9]. The causes of this are presently uncertain, but are consistent with a preferential involvement of aerobically-inefficient fast-twitch muscle fibers in the exercise [26]. Moreover, during FES cycling, only a subset of the muscle groups utilized in voluntary cycling are stimulated, and in a manner which is necessarily suboptimal with respect to natural patterns of recruitment.
The disturbance test involves large changes in total power output. These changes simulate typical changes in load which would occur during mobile cycling, as a result of external influences such as slope or wind disturbances. The good control which is maintained during the disturbance test illustrates the utility of the control approach for recreational cycling. However, the results here are obtained with the tricycle on a trainer, where the subject's total body mass and dynamic inertia have little effect on the plant dynamics (on the trainer, only the legs are involved). During mobile cycling the total body inertia will dominate - thus, we would expect to see significantly different motor-cadence dynamics in this situation. Although the overall design methodology would be the same, the possible effects on performance must be investigated in future work.

We suggest that electric motor assist could also be highly useful during the initial training phase for new cyclists. In the initial stages of a cycle training program, a subject can typically cycle for only a very short time, with tolerance having to be built up gradually over a period of time. With motor assist, on the other hand, the legs can be cycled for arbitrarily long periods of time, and, therefore, the legs can be stimulated and trained during actual FES cycling for longer periods (at an appropriate level of stimulation, to ensure that over-stimulation and subsequent damage to muscle fibers does not occur). This may prove to be a much more effective training protocol.

Finally, we note that the identification and control design approaches utilized here are straightforward, rely on standard methods, and can be quickly implemented. During the feedback control tests the complete system identification and control design procedures were carried out at the start of each experimental session. However, we have since determined that a single, fixed-parameter linear controller (obtained during identification and control design in some session on some day) will give satisfactory performance on subsequent sessions on different days over a considerable time period and, indeed, for different subjects. In this respect, we note that new system identification experiments were not performed on the days on which the exercise tests reported above were carried out; predesigned controllers from previous control-testing sessions were used. The stimulation power control loop nevertheless performed well (Figs. 12 and 13), indicating an underlying robustness of the control designs. Thus, it is not necessary to carry out new identification and control tests at each cycling session.

\section{CONCLUSION}

The integrated control strategy is effective in facilitating FES exercise testing under conditions of well-controlled cadence and power output. Our control approach significantly extends both the range and the exercise-test sensitivity for FES cycling and should, thus, allow more stringent characterization of physiological response profiles and, therefore, estimation of key parameters of aerobic function (such as peak oxygen uptake, the lactate threshold, work efficiency, and the $\dot{\mathrm{VO}}_{2}$ time constant). This represents a substantial advance in the SCI population where the maximal exercise workrate is typically substantially compromised. 
We further conclude that the addition of electric motor assist, with an integrated feedback control strategy, can significantly improve the overall performance of mobile recreational cycling. It can compensate for external disturbances (including slope, wind resistance and loss of muscle power), and it can greatly increase the overall power output, thereby significantly extending the range of operation.

\section{ACKNOWLEDGMENT}

The authors are grateful to the subjects who participated in the study.

\section{REFERENCES}

[1] R. Glaser, J. Bruner, S. Feinberg, and S. Collins, "Locomotion via paralyzed leg muscles, feasibility study for a leg propelled vehicle," J. Rehab. Res. Dev., vol. 20, pp. 53-61, 1983.

[2] J. Petrofsky, H. Heaten, and C. Phillips, "Outdoor bicycle for exercise in paraplegics and quadriplegics," J. Biomed. Eng., vol. 5, pp. 292-296, Oct. 1983.

[3] D. J. Pons, C. L. Vaughan, and G. G. Jaros, "Cycling device powered by the electrically stimulated muscles of paraplegics," Med. Biol. Eng. Comput., vol. 27, pp. 1-7, Jan. 1989.

[4] M. Gföhler, M. Loicht, and P. Lugner, "Exercise tricycle for paraplegics," Med. Biol. Eng. Comput., vol. 36, pp. 118-121, 1998.

[5] T. Perkins, N. Donaldson, R. Fitzwater, G. Phillips, and D. E. Wood, "Leg powered paraplegic cycling system using surface functional electrical stimulation," in Proc. 7th Int. Workshop Functional Electrical Stimulation, Vienna, Austria, 2001

[6] K. J. Hunt, T. Schauer, N.-O. Negård, W. Stewart, and M. H. Fraser, "A pilot study of lower-limb FES cycling in paraplegia," in Proc. 7th Annu. Conf. Int. Functional Electrical Stimulation Soc., Ljubljana, Slovenia, 2002.

[7] T. Janssen, R. Glaser, and D. Shuster, "Clinical efficacy of electrical stimulation exercise training: Effects on health, fitness, and function," Top. Spinal Cord Injury Rehab., vol. 3, no. 3, pp. 33-49, 1998.

[8] N. Lamarra, B. J. Whipp, S. A. Ward, and K. Wasserman, "Effect of interbreath fluctuations on characterizing exercise gas-exchange kinetics," J. Appl. Physiol., vol. 62, pp. 2003-2012, 1987.

[9] J. Roca and B. J. Whipp, Eds., Clinical Exercise Testing. Sheffield, U.K.: European Respiratory Journals, 1997, vol. 2, European Respiratory Monographs.

[10] K. Wasserman, J. E. Hansen, D. Y. Sue, R. Casaburi, and B. J. Whipp, Principles of Exercise Testing and Interpretation. Philadelphia, PA: Lippincott, Williams, and Wilkins, 1999.

[11] E. F. Coyle, L. S. Sidosis, J. F. Horowitz, and J. D. Belttz, "Cycling efficiency is related to the percentage of type I muscle fibers," Med. Sci. Sports Exer, vol. 24, pp. 782-788, 1992.

[12] D. Theisen, C. Fornusek, J. Raymond, and G. M. Davis, "External power output changes during prolonged cycling with electrical stimulation," $J$. Rehab. Med., vol. 34, pp. 171-175, 2002.

[13] J. Petrofsky and J. Smith, "Three wheel cycle ergometer for use by men and women with paralysis," Med. Biol. Eng. Comput., vol. 30, pp. 364-369, 1992.

[14] P. Eser and N. Donaldson, "Influence of different stimulation frequencies on power output during FES-cycling in recently injured SCI people," in Proc. 7th Ann. Conf. Int. Functional Electrical Stimulation Soc., Ljubljana, Slovenia, 2002.

[15] J. Petrofsky and R. Stacy, "The effect of training on endurance and the cardiovascular responses of individuals with paraplegia during dynamic exercise induced by functional electrical stimulation," Eur. J. Appl. Physiol., vol. 64, pp. 487-492, 1992.

[16] G. F. Phillips, J. R. Adler, and S. J. G. Taylor, "A portable programmable eight-channel surface stimulator," in Proc. Ljubljana FES Conf., 1993, pp. 166-168.

[17] W. L. Beaver, K. Wasserman, and B. J. Whipp, "On-line computer analysis and breath-by-breath graphical display of exercise function tests," J. Appl. Physiol., vol. 34, pp. 128-132, 1973.

[18] L. Ljung, System Identification: Theory for the User, 2nd ed. Englewood Cliffs, NJ: Prentice-Hall, 1999, Information and System Sciences Series.
[19] K. J. Åström and B. Wittenmark, Computer Controlled Systems, 3rd ed. Englewood Cliffs, NJ: Prentice-Hall, 1997.

[20] B. J. Whipp, S. A. Ward, N. Lamarra, J. A. Davis, and K. Wasserman, "Parameters of ventilatory and gas exchange dynamics during exercise," J. Appl. Physiol., vol. 52, pp. 1506-1513, 1982.

[21] B. J. Whipp, J. A. Davis, F. Torres, and K. Wasserman, "A test to determine the parameters of aerobic function during exercise," J. Appl. Physiol., vol. 50, pp. 217-221, 1981.

[22] B. J. Whipp and F. Ozyener, "The kinetics of exertional oxygen uptake: Assumptions and inferences," Medicina Dello Sport, vol. 51, pp. 139-149, 1998.

[23] T. J. Barstow, A. M. E. Scremin, D. L. Mutton, C. F. Kunkel, T. G. Cagle, and B. J. Whipp, "Gas exchange kinetics during functional electrical stimulation in subjects with spinal cord injury," Med. Sci. Sports Exer., vol. 27, pp. 1284-1291, 1995.

[24] _ - "Changes in gas-exchange kinetics with training in patients with spinal cord injury," Med. Sci. Sports Exer., vol. 28, pp. 1221-1228, 1996.

[25] R. M. Glaser, S. F. Figoni, S. P. Hooker, M. M. Rodgers, B. N. Ezenwa, A. G. Suryaprasad, S. C. Gupta, and T. Mathews, "Efficiency of FNS leg cycle ergometry," in Proc. 11th Annu. Conf. IEEE Engineering Medicine Biology. Soc., 1989, pp. 961-963.

[26] M. T. Crow and M. J. Kushmerick, "Chemical energetics of slowand fast-twitch muscles of the mouse," J. Gen. Physiol., vol. 79, pp. 147-166, 1982.

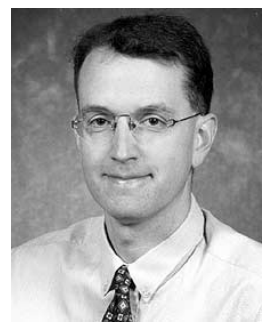

Ken Hunt (S'87-M'87) received the B.Sc. degree in electrical and electronic engineering (first-class honors) and the Ph.D. degree in control theory from the University of Strathclyde, Glasgow, U.K., in 1984 and 1987, respectively.

From 1989 to 1992, he held a Royal Society of Edinburgh Personal Research Fellowship, and from 1992 to 1997, he was a Research Scientist and Project Leader with Daimler-Benz Systems Technology Research, Berlin, Germany. He is currently a Wylie Professor of Mechanical Engineering and the Director of the Centre for Rehabilitation Engineering, Glasgow University, Glascow, Scotland, U.K. He is also a Clinical Research Professor with the National Spinal Injuries Unit, Southern General Hospital, Glascow. His research interests include feedback control of balance in paraplegia, lower-limb cycling in paraplegia, and assisted upper-limb arm-cranking exercise in tetraplegia.

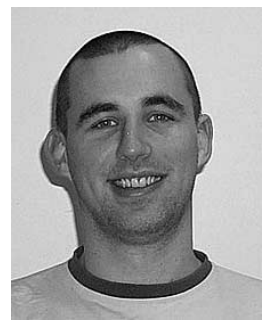

Barry Stone is from Inverness, Scotland. He received the degree in mechanical engineering (first-class honors) from the University of Glasgow, Glasgow, U.K., in 2001. He is working toward the $\mathrm{Ph} . \mathrm{D}$. degree at the same university.

He currently works in the Centre for Rehabilitation Engineering, University of Glasgow. His research interests include functional electrical stimulation exercise for paraplegics and robust control engineering.

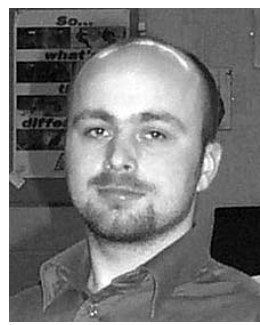

Nils-Otto Negård was born in Tynset, Norway, in 1975. He received the degree in engineering cybernetics from the Norwegian University of Science and Technology, Trondheim, Norway, in 2000. He is working toward the Ph.D. degree in electrical engineering at the University of Magdeburg, Magdeburg, Germany.

From 2000 to 2002, he was a Research Assistant with the Centre for Systems and Control, University of Glasgow, Glasgow, U.K. Currently, he is a Research Scientist at the Max Planck Institute for Dynamics of Complex Technical Systems, Magdeburg. His research interests include nonlinear control of FES-assisted gait in stroke patients and FES-cycling. 




Thomas Schauer was born in Magdeburg, Germany, in 1974. He received the Dipl.-Ing. degree in electrical engineering from University of Magdeburg, Magdeburg, in 1997. He was working toward the Ph.D. degree from 1998-2001 at the Centre for Systems and Control, the University of Glasgow, Glasgow, U.K., in 2001.

Currently, he is a Research Scientist and Project Leader in the Systems and Control Theory Group, the Max Planck Institute for Dynamics of Complex Technical Systems, Magdeburg. His research interests include the control of paralyzed human extremities, functional electrical stimulation, and the modeling of neuro-muscular systems.

Dr. Schauer is a member of the IEEE Engineering in Medicine and Biology Society and the International Functional Electrical Stimulation Society.

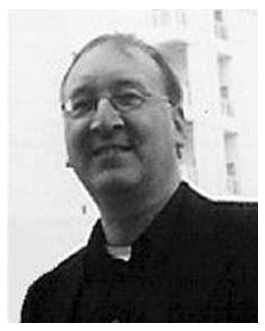

Matthew H. Fraser is a Consultant in spinal-cord injury at the Queen Elizabeth National Spinal Injuries Unit, Southern General Hospital, Glasgow, U.K.

Dr. Fraser is a Fellow of the Royal College of Surgeons (FRCS).

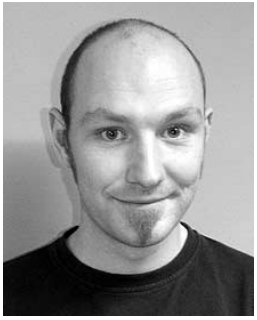

Andrew J. Cathcart received the degree in physiology and sports science (first class honors) from the University of Glasgow, Glasgow, U.K., in 1999. $\mathrm{He}$ is currently working toward the Ph.D. degree at the Centre for Exercise Science and Medicine (CESAME), the University of Glasgow.

He recently finished a six-month appointment as an Associate Lecturer at the University of Glasgow. His research interests include the fields of physiological control mechanisms and exercise-based rehabilitation strategies.

Mr. Cathcart received the Hunter medal for excellence from the University of Glasgow in 1999.

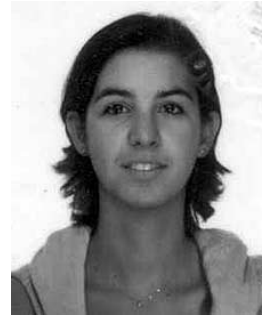

Chiara Ferrario was born in Milano, Italy, in 1977. She received the M.Sc. degree in biomedical engineering from the Polytechnic of Milano, Milano, in 2002. She is working toward the Ph.D. degree at the University of Glasgow, Glasgow, U.K., where she works in the Centre for Rehabilitation Engineering.

Her research interests include functional electrical stimulation exercise for paraplegic people and energy expenditure during exercise.

Susan A. Ward received the D.Phil. degree in physiology from Oxford University, Oxford, U.K., in 1974.

Currently, she is a Professor of sport and exercise science at the University of Leeds, Leeds, U.K. Following two years as a Lecturer in the Department of Physiology, Liverpool University, Liverpool, U.K., she moved to the University of California at Los Angeles, becoming Professor of Anesthesiology and Physiology in 1988. She returned to the U.K. in 1993, first to the Department of Physiology, the University of London's St George's Hospital Medical School, London, U.K., then to South Bank University, London, as Chair of Sports Science and, in 1998, to the University of Glasgow, Glascow, U.K., as Director of the Centre for Exercise Science and Medicine. In 2003, she was appointed to the Chair of Sport and Exercise Science and Head of the School of Sport and Exercise Sciences, the University of Leeds. She is a Principal Editor of the European Journal of Applied Physiology and Experimental Physiology. Her research interests include the control of ventilation, pulmonary gas exchange and muscle energetics during exercise in health and disease. She has authored over 110 publications on these topics.

Dr. Ward is a Fellow of the American College of Sports Medicine and of the European College of Sports Science. She currently serves as Treasurer of the European College of Sports Science, and Secretary of the Pulmonary Circulation, Gas Exchange, and Exercise Group of the European Respiratory Society.

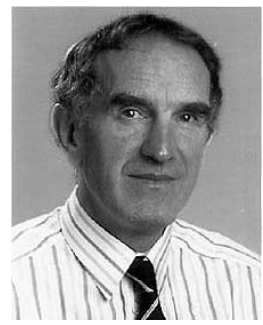

Stan Grant is currently a Senior Lecturer in Exercise Science with the Institute of Biomedical and Life Sciences, the University of Glasgow, Glasgow, U.K. $\mathrm{He}$ is also an exercise consultant to Glasgow Celtic Football Club, Glasgow. He has particular expertise in the testing of athletes (particularly soccer players and climbers) and the evaluation of training methods. His other research interests include the monitoring of physiological responses in a range of environmental conditions including cold and hypoxia, and the measurement of physical activity in preschool aged children. He has over 70 publications in exercise-related areas.

Dr. Grant is a member of the advisory panel to the Scottish Institute of Sports Medicine and Sports Science. 